OPEN ACCESS

Edited by:

Tong Liu,

Tianjin Medical University, China

Reviewed by:

Deyong Long,

Capital Medical University, China

Ohad Ziv,

Case Western Reserve University,

United States

${ }^{*}$ Correspondence: Yunlong Xia

yunlong_xia@126.com

†These authors have contributed equally to this work

Specialty section:

This article was submitted to

Cardiac Rhythmology,

a section of the journal

Frontiers in Cardiovascular Medicine

Received: 01 March 2021

Accepted: 06 May 2021

Published: 08 June 2021

Citation:

Zhang $R$, Wang $Y$, Yang $M$, Yang $Y$ Wang Z, Yin X, Dong Y, Yu X, Xiao X, Gao $L$ and Xia $Y$ (2021) Risk Stratification for Atrial Fibrillation and Outcomes in Tachycardia-Bradycardia Syndrome: Ablation vs. Pacing

Front. Cardiovasc. Med. 8:674471. doi: 10.3389/fcrm.2021.674471

\section{Risk Stratification for Atrial Fibrillation and Outcomes in Tachycardia-Bradycardia Syndrome: Ablation vs. Pacing}

\author{
Rongfeng Zhang ${ }^{\dagger}$, Yue Wang ${ }^{\dagger}$, Minghui Yang ${ }^{\dagger}$, Yiheng Yang, Zhengyan Wang, \\ Xiaomeng Yin, Yingxue Dong, Xiaohong Yu, Xianjie Xiao, Lianjun Gao and Yunlong Xia*
}

Department of Cardiology, First Affiliated Hospital of Dalian Medical University, Dalian, China

Background: Catheter ablation of atrial fibrillation is an alternative treatment for patients with tachycardia-bradycardia syndrome (TBS) to avoid pacemaker implantation. The risk stratification for atrial fibrillation and outcomes between ablation and pacing has not been fully evaluated.

Methods: This retrospective study involved 306 TBS patients, including 141 patients who received catheter ablation (Ablation group, age: $62.2 \pm 9.0$ months, mean longest pauses: $5.2 \pm 2.2 \mathrm{~s}$ ) and 165 patients who received pacemaker implement (Pacing group, age: $62.3 \pm 9.1$ months, mean longest pauses: $6.0 \pm 2.3 \mathrm{~s})$. The primary endpoint was a composite of call cause mortality, cardiovascular-related hospitalization or thrombosis events (stroke, or peripheral thrombosis). The second endpoint was progress of atrial fibrillation and heart failure.

Results: After a median follow-up of 75.4 months, the primary endpoint occurred in significantly higher patients in the pacing group than in the ablation group (59.4 vs.15.6\%, OR 6.05, 95\% Cl: 3.73-9.80, $P<0.001)$. None of deaths was occurred in ablation group, and 1 death occurred due to cancer. Cardiovascular-related hospitalization occurred in $50.9 \%$ of the pacing group compared with $14.2 \%$ in the ablation group (OR: 4.87 , 95\% Cl: 2.99-7.95, $P<0.001)$. More thrombosis events occurred in the pacing group than in the ablation group (12.7 vs. $2.1 \%$, OR 6.06, 95\% Cl: $1.81-20.35, P=0.004$ ). Significant more patients progressed to persistent atrial fibrillation in pacing group than in ablation group ( 23.6 vs. $2.1 \%, P<0.001)$. The NYHA classification of the pacing group was significantly higher than that of the ablation group $(2.11 \pm 0.83$ vs. $1.50 \pm 0.74$, $P<0.001)$. The proportion of antiarrhythmic drugs and anticoagulants used in the pacing group was significantly higher than that in the ablation group (41.2 vs. $7.1 \%, P<0.001$; 16.4 vs. $2.1 \%, P=0.009$ ).

Conclusion: Catheter ablation for patients with TBS was associated with a significantly lower rate of a composite end point of cardiovascular related hospitalization and thromboembolic events. Furthermore, catheter ablation reduced the progression of atrial fibrillation and heart failure.

Keywords: atrial fibrillation, tachycardia-bradycardia syndrome, long pauses, catheter ablation, pacing, long outcome 


\section{INTRODUCTION}

Tachycardia-bradycardia syndrome (TBS) is a common clinical arrhythmia used to describe a special subtype of sick sinus syndrome (SSS), with a long pause (RR intervals $>3 \mathrm{~s}$ ) on termination of atrial fibrillation (AF) $(1,2)$. Patients with TBS are at a higher risk of amaurosis, syncope, and even sudden death (3). Guidelines determined that catheter ablation could be used as an alternative treatment for patients with TBS to avoid pacemaker implantation and the evidence recommendation level is IIa $(4,5)$. However, both treatment options for patients are at potential risk. AF or device related problems may remain even after pacemaker implantation, such as (1) the effect of antiarrhythmic drugs on atrial fibrillation is very limited and the incidences of arrhythmic effects and extracardiac adverse effects are high (6); (2) the incidence of atrial fibrillation-related symptoms, rehospitalization, stroke, progression of atrial fibrillation, and atrial fibrillation-mediated cardiomyopathy persists (7-9); and (3) pacemaker-related complications, such as infections and pacemaker-mediated cardiomyopathy are issues (10).

Previous studies only compared the feasibility and safety between ablation and pacing strategy in TBS patients. Studies showed that $>85 \%$ of the patients may avoid the pacemaker when ablation for atrial fibrillation was performed. However, the long outcome of ablation for atrial fibrillation superior to pacing is not clear. In our study, we conducted a large-scale retrospective analysis involving 306 patients with TBS with an average follow-up time of 6 years, to evaluate whether catheter ablation improved the long-term outcome of the patients with TBS compared with cardiac pacing.

\section{METHOD}

\section{Study Population}

This single-center retrospective study was approved by the Ethics Committee of the First Affiliated Hospital of Dalian Medical University, Liaoning Province, China. We retrospectively analyzed 1,371 patients undergoing pacemaker implantation and 795 patients underwent catheter ablation due to atrial fibrillation from 2012 to 2017. A total of 306 patients with TBS were ultimately selected, including 165 patients with pacemaker implantation (Pacing group) and 141 patients with catheter ablation (Ablation group).

In this study, TBS diagnosis was in accordance with the diagnostic criteria of BM Kaplan (11), which define TBS as paroxysmal atrial fibrillation, flutter, or tachycardia followed by sinoatrial block or sinus arrest resulting in Stokes-Adams attacks. Patients who had both atrioventricular block/structural heart disease/heart failure, and/or had received radiofrequency ablation or pacemaker implantation in the past were excluded from the study (the screening process is shown in Figure 1).

Abbreviations: AAD, Antiarrhythmic drug; AVN, Atrioventricular node; AF, Atrial fibrillation; AUC, Area-under-the-curve; CI, Confidential interval; HF, Heart failure; LAD, Left atrial diameter; LVD, Left ventricular diameter; LVEF, Left ventricular ejection fraction; NOAC, New oral anticoagulants; NYHA, New York Heart Association; TBS, Tachycardia-bradycardia syndrome; Vit K, vitamin K.

\section{Operation Strategy}

We provided two treatment options and listed the pros and cons to the patient prior to procedure. And then the patient chose one strategy. In the ablation group, the TBS patients were all diagnosed with paroxysmal atrial fibrillation and received pulmonary vein isolation (PVI) only without addition lesion sets as the ablation strategy. The ablation procedures for this group are as described in a previous study (12). In brief, PV isolation was performed by ablation catheter Navistar Thermocool 3.5mm D-F curve with Smart Touch technology (Biosense Webster) using contiguous circumferential lesions guided by (lasso ${ }^{\mathrm{TM}}$, Biosense and Webster, Inc., CA, USA). RF energy was applied in a power-controlled mode with a power limited of $35 \mathrm{~W}$ ( $30 \mathrm{~W}$ at the posterior wall) and a maximal temperature of $45^{\circ} \mathrm{C}$. At each point, a radiofrequency current was applied until a voltage of $<0.1 \mathrm{mV}$ was achieved, with a maximum of $30 \mathrm{~s}$ per point. In the pacing group, the TBS patients all had paroxysmal atrial fibrillation and received DDD or DDDR pacing, and the procedures were as described by Dong et al. (13).

\section{Study Endpoint}

The primary endpoint of this study was a composite endpoint, consisting of all cause mortality cardiovascular rehospitalization and thromboembolic events. Cardiovascular rehospitalization was defined as patients who were re-hospitalized for cardiovascular diseases, including tachycardia, bradycardia, coronary atherosclerotic heart disease (i.e., angina pectoris and/or myocardial infarction), and heart failure. The definition of thrombotic events referred to the occurrence of stroke and/or peripheral thrombotic events (i.e., pulmonary embolism, mesenteric artery embolism, and lower extremity arterial embolism). The definition of the progression of heart failure, we are mainly concerned with NYHA cardiac function grade and left ventricular enlargement or ejection fraction decrease.

\section{Follow-Up}

Patients in the ablation group and the pacing group underwent follow-up for an average of $73.2 \pm 17.0$ months and $77.6 \pm$ 21.3 months, respectively. The follow-ups were completed by a designated follow-up clinic. Patients in the ablation group had follow-ups in the postoperative months 3,6 , and 12 , followed by once every 12 months after the operation via telephone and outpatient visit. Patients in the pacing group were followed up by retrospectively reviewing patient pacemaker programmed records, as well as through telephone and outpatient visits. Twenty-four-hour holter or ECG in the ablation group and pacing group were performed to detect the recurrence of $\mathrm{AF}$ at 3, 6, and 12 months visit and annually visit during follow up period. Patient data such as symptoms, recurrence of atrial fibrillation, repeated pacing or catheter ablation, usage of medication, rehospitalization occurrence, and the reasons for cardiovascular rehospitalization or thromboembolic events were collected during follow-ups.

\section{Statistical Analysis}

The continuous variables are presented as mean \pm standard deviation and compared using an independent sample $t$-test, and 

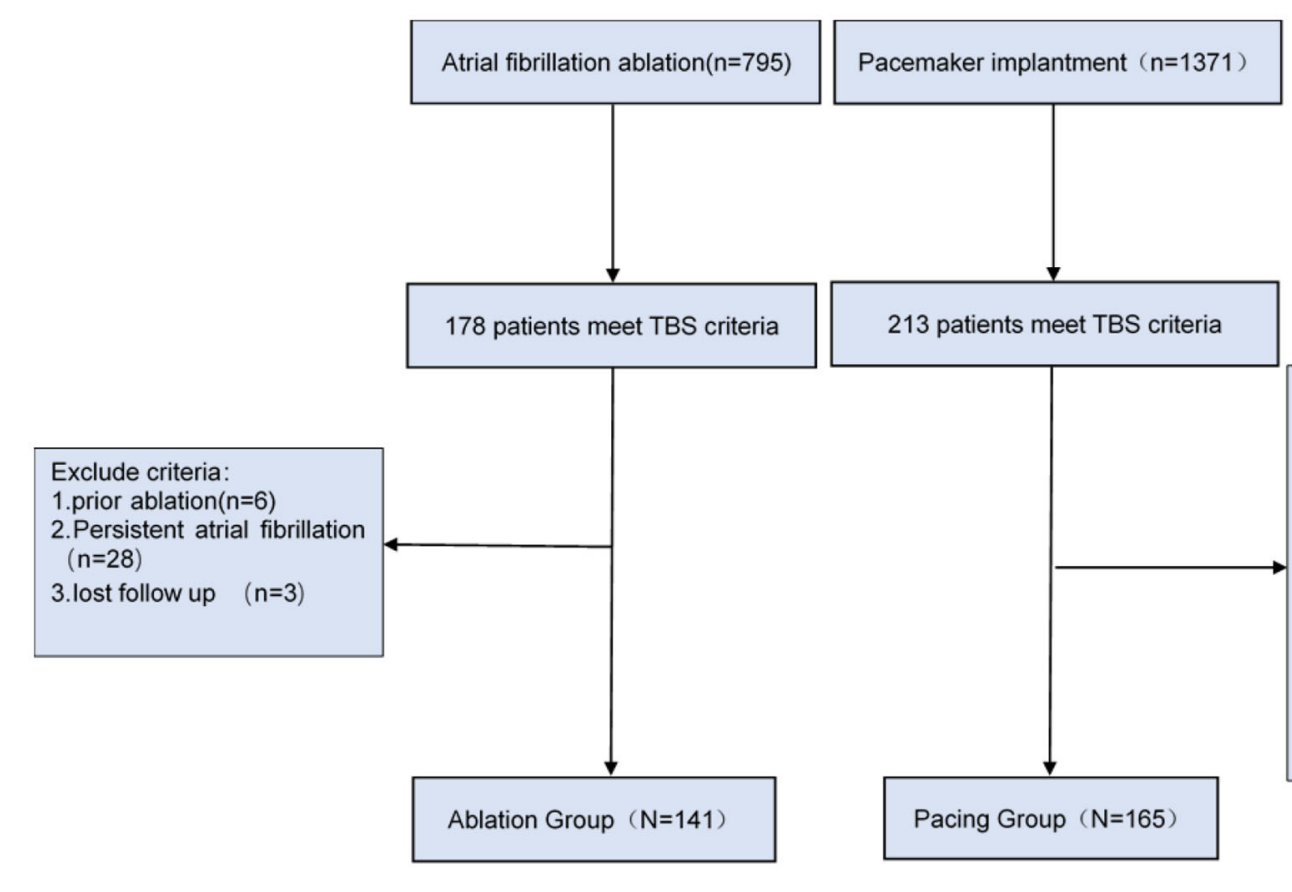

Exclude criteria:

1. Combined with atrioventricular block $(n=24)$

2. Structural heart disease $(n=10)$

3. Prior ablation or pacemaker implement $(n=2)$

4. Heart Failure $(n=6)$

5. prior diagnosis as sick sinus syndrome $(n=3)$

6.lost follow up $(n=3)$

FIGURE 1 | Study population and flow chart.

the categorical variables are presented as count and percentage and analyzed using the chi-square test or odds ratio $(\mathrm{OR})$ value. $P$ $<0.05$ was considered to indicate a significant difference between the groups. The Kaplan-Meier curve was used to compare the incidences of cardiovascular-related rehospitalization, stroke, and/or peripheral thromboembolism, and the log-rank test was used for evaluation. SPSS 23.0 software (IBM Corp., Armonk, NY, USA) was used for statistical analysis in this study.

\section{RESULTS}

\section{General Characteristics of the Study Subjects}

The clinical characteristics of the patients with TBS are shown in Table 1. A total of 306 patients with TBS were selected, including 141 patients in the ablation group, with women accounting for $53.2 \%$ and an average age of $62.7 \pm 8.8$ years, and 165 patients in the pacing group, with women accounting for $52.7 \%$ and an average age of $62.4 \pm 8.4$ years. The longest pauses after termination of atrial fibrillation in the pacing group was slightly longer than that in the ablation group, but this difference was not significant $(6.0 \pm 2.4$ vs. $5.2 \pm 2.2 \mathrm{~s}, P=0.081)$. The total heart rate per $24 \mathrm{~h}$ of the pacing group were lower than that of the ablation group $(89,311 \pm 19,422$ vs. $97,179 \pm 16,888, P=$ $0.030)$, but the average heart rate had no statistically significant difference between the two groups $(P=0.283)$. There was no significant difference in CHA2DS2-VASc score between the two groups ( $1.65 \pm 1.0$ vs. $1.75 \pm 1.2, P=0.469)$ (Table 2$)$.

\section{Comparison of Therapeutic Results Between Ablation Group and Pacing Group} After an average follow-up of $75.5 \pm 19.1$ months, 116 patients $(82.3 \%)$ in the ablation group maintained sinus rhythm. In addition, 16 patients (11.4\%) in the ablation group had pacemaker implantation due to recurrence of atrial fibrillation with long pauses, and another 6 patients (4.3\%) in the ablation group had recurring atrial fibrillation, but no long pauses and without pacemaker implantation. In the pacing group, only 31 patients (18.8\%) maintained sinus rhythm, and 8 patients (4.8\%) received ablation. Compared with the ablation group, more patients in the pacing group progressed to persistent atrial fibrillation [39 (23.6\%) vs. $3(2.1 \%), P<0.001]$, and more patients used antiarrhythmic drugs and anticoagulants [68 (41.2\%) vs. 10 (7.1\%), $P<0.001]$ and [27 (16.4\%) vs. $3(2.1 \%), P<$ 0.001]. The New York Heart Association (NYHA) functional classification grade of the pacing group was significantly higher than that of the ablation group $(2.11 \pm 0.83$ vs. $1.50 \pm 0.74, P<$ 0.001). According to $\mathrm{CHA}_{2} \mathrm{DS}_{2}$-VASc score, 65 patients in pacing group need long-term anticoagulation therapy, but only 27 people actually insist on oral anticoagulation. 5 patients stopped oral anticoagulants due to bleeding events, 28 patients stopped taking anticoagulants without authorization, and 5 patients took anticoagulants irregularly. Fifty-nine people in ablation group need anticoagulation, but only 3 patients actually insist on oral anticoagulation. Atrial fibrillation was cured in 50 patients, 4 patients personally stopped taking anticoagulants, and 2 patients took anticoagulants irregularly. No significant difference in the incidence of surgery-related complications was found between the ablation and pacing groups $(P>0.05$; Table 3$)$. 
TABLE 1 | Characteristics of the study subjects.

\begin{tabular}{lc}
\hline & TBS patients $(\boldsymbol{n}=\mathbf{3 0 6})$ \\
\hline Female $(n, \%)$ & $162(52.9 \%)$ \\
Age (mean \pm SD, y) & $62.6 \pm 8.6$ \\
Diabetes $(n, \%)$ & $75(24.5 \%)$ \\
Hypertension $(n, \%)$ & $137(44.7 \%)$ \\
Coronary heart disease $(n, \%)$ & $61(19.9 \%)$ \\
Stroke ( $n$, \%) & $5(1.6 \%)$ \\
AF duration (Mean \pm SD, y) & $4.6 \pm 3.4$ \\
Total heart rate (mean \pm SD, beats/24h) & $91,486 \pm 13,341$ \\
Mean heart rate (mean \pm SD, beats/min) & $64.9 \pm 8.4$ \\
Longest pause (mean \pm SD, s) & $5.6 \pm 2.3$ \\
Symptom & \\
Amaurosis $(n, \%)$ & $111(36.2 \%)$ \\
Syncope $(n, \%)$ & $97(31.7 \%)$ \\
LAD (mean \pm SD, mm) & $38.4 \pm 4.18$ \\
LVD (mean \pm SD, mm) & $45.42 \pm 4.16$ \\
LVEF (mean \pm SD, \%) & $57.7 \pm 2.38$ \\
CHA $2 S_{2}$-VASc score (14) & $1.7 \pm 1.1$ \\
NYHA classification (mean \pm SD) & $1.4 \pm 0.5$ \\
Ablation therapy $(n, \%)$ & $141(46 \%)$ \\
Pacing therapy $(n, \%)$ & $165(54 \%)$ \\
Outcomes & \\
Cardiovascular related hospitalization $(n, \%)$ & $104(34.0 \%)$ \\
Stroke $(n, \%)$ & $18(5.9 \%)$ \\
Peripheral thrombosis $(n, \%)$ & $6(2.0 \%)$ \\
\hline
\end{tabular}

\section{Comparison of Endpoint Between Ablation Group and Pacing Group}

Compared with the ablation group, the pacing group had a higher incidence of the primary study endpoint (59.4 vs.14.2\%, OR 6.05, 95\% CI: 3.73-9.80, $P<0.001)$. The risk of cardiovascular related hospitalization in the pacing group was 4.87 -fold that of the ablation group (95\% CI: 3.57-11.01, $P<0.001$ ). None of deaths was occurred in ablation group, and 1 death occurred due to cancer. A total of 84 cardiovascular-related hospitalization events occurred in the pacing group, primarily due to tachycardia (35.7\%), heart failure (27.7\%), coronary heart disease $(4.2 \%)$. There were only 20 cardiovascular-related hospitalization events occurred in the ablation group, which were primarily due to bradycardia $(7.8 \%)$, tachycardia $(2.1 \%)$, heart failure $(2.1 \%)$, and coronary heart disease (2.1\%). A total of 9 patients (5.4\%) hospitalized due to tachycardia underwent cardioversion therapy. The risk of thromboembolic events in the pacing group was 6.06-fold that of the ablation group (95\% CI: 1.81-20.35, $P<0.001$ ). A total of 15 strokes and 6 peripheral vascular embolization occurred in the pacing group, while only 3 strokes occurred in the ablation group (Table 4, Figures 2-4). After correcting hypertension, diabetes, stroke history, anticoagulation and CHA2DS2-VASc score by cox regression, there was still significant difference in Primary end point between the two groups $(p<0.001)$.
TABLE 2 | Characteristics of the two groups.

\begin{tabular}{|c|c|c|c|}
\hline & $\begin{array}{l}\text { Ablation group } \\
\qquad(n=141)\end{array}$ & $\begin{array}{l}\text { Pacing group } \\
\qquad(n=165)\end{array}$ & $P$ \\
\hline Female $(n, \%)$ & 75 (53.2\%) & 87 (52.7\%) & 0.935 \\
\hline Age (mean, y) & $62.7 \pm 8.8$ & $62.4 \pm 8.4$ & 0.790 \\
\hline Diabetes (n, \%) & $30(21.3 \%)$ & $45(27.3 \%)$ & 0.224 \\
\hline Hypertension $(n, \%)$ & 60 (42.6\%) & 77 (46.7\%) & 0.471 \\
\hline Coronary heart disease $(n, \%)$ & 24 (17.0\%) & 37 (20.6\%) & 0.238 \\
\hline Stroke $(n, \%)$ & $2(1.4 \%)$ & $3(1.8 \%)$ & 0.783 \\
\hline $\begin{array}{l}\text { Total heart rate (mean } \pm S D \text {, } \\
\text { beats/24 h) }\end{array}$ & $97,179 \pm 16,888$ & $89,311 \pm 19,422$ & 0.030 \\
\hline $\begin{array}{l}\text { Mean heart rate (mean } \pm \mathrm{SD} \text {, } \\
\text { beats/min) }\end{array}$ & $65 \pm 7$ & $64 \pm 8$ & 0.283 \\
\hline AF duration (mean $\pm \mathrm{SD}, \mathrm{y}$ ) & $4.3 \pm 2.96$ & $5.0 \pm 3.78$ & 0.065 \\
\hline Longest pause (mean $\pm \mathrm{SD}, \mathrm{s}$ ) & $5.2 \pm 2.2$ & $6.0 \pm 2.3$ & 0.081 \\
\hline \multicolumn{4}{|l|}{ Symptom } \\
\hline Amaurosis (n, \%) & 47 (33.3\%) & 64 (38.8\%) & 0.323 \\
\hline Syncope (n, \%) & $43(30.5 \%)$ & $54(32.7 \%)$ & 0.676 \\
\hline $\begin{array}{l}\mathrm{CHA}_{2} \mathrm{DS}_{2} \text {-VASc score (mean } \pm \\
\mathrm{SD} \text { ) }\end{array}$ & $1.65 \pm 1.0$ & $1.75 \pm 1.2$ & 0.469 \\
\hline NYHA classification (mean \pm SD) & $1.37 \pm 0.48$ & $1.45 \pm 0.49$ & 0.130 \\
\hline LAD (mean \pm SD, mm) & $37.96 \pm 3.91$ & $38.78 \pm 4.37$ & 0.086 \\
\hline LVD (mean \pm SD, mm) & $45.18 \pm 3.86$ & $45.45 \pm 4.05$ & 0.553 \\
\hline LVEF (mean \pm SD, \%) & $57.95 \pm 2.58$ & $57.52 \pm 2.17$ & 0.116 \\
\hline
\end{tabular}

TABLE 3 | Comparison of therapeutic results between ablation group and pacing group.

$\begin{array}{ccc}\begin{array}{c}\text { Ablation group } \\ (n=141)\end{array} & \text { Pacing group } & P \\ (n=165) & \end{array}$

\begin{tabular}{lccc}
\hline Symptoms & & & \\
$\quad$ Amaurosis $(n, \%)$ & $9(6.3 \%)$ & 0 & NS \\
$\quad$ Syncope $(n, \%)$ & $7(4.9 \%)$ & 0 & NS \\
Freedom from AF $(n, \%)$ & $116(82.3 \%)$ & $31(18.8 \%)$ & $<0.001$ \\
AF progression $(n, \%)$ & $3(2.1 \%)$ & $39(23.6 \%)$ & $<0.001$ \\
Heart failure progression $(n, \%)$ & $4(2.8 \%)$ & $18(10.9 \%)$ & 0.006 \\
NYHA class (mean \pm SD) & $1.50 \pm 0.74$ & $2.11 \pm 0.83$ & $<0.001$ \\
AADs use $(n, \%)$ & $10(7.1 \%)$ & $68(41.2 \%)$ & $<0.001$ \\
Anticoagulation $(n, \%)$ & $3(2.1 \%)$ & $27(16.4 \%)$ & $<0.001$ \\
Crossover therapy & 16 & 8 & 0.035 \\
$\quad$ Pacemaker implement $(n, \%)$ & $16(11.3 \%)$ & - & NS \\
Cather ablation $(n, \%)$ & - & $8(4.8 \%)$ & NS \\
Operation complications $(n, \%)$ & $2(1.4 \%)$ & $4(2.4 \%)$ & 0.527 \\
\hline
\end{tabular}

NS, None Significant.

\section{DISCUSSION}

\section{Main Research Findings}

This large-scale retrospective study involved 306 patients with TBS, including 141 patients in the ablation group and 165 patients in the pacing group, with an average follow-up of nearly 6 years. The use of ablation for atrial fibrillation in TBS patients was associated with a significantly lower rate of a composite of cardiovascular hospitalization and thrombosis than pacing 
TABLE 4 | Comparison of the primary end point between ablation group and pacing group.

\begin{tabular}{|c|c|c|c|c|c|}
\hline & $\begin{array}{c}\text { Ablation group } \\
\qquad N(\%)\end{array}$ & $\begin{array}{c}\text { Pacing group } \\
\quad N(\%)\end{array}$ & HR $(95 \% \mathrm{Cl})$ & $P$ & $P$-adj \\
\hline Primary end point & $20(14.2 \%)$ & 98 (59.4\%) & $6.05(3.73-9.80)$ & $<0.001$ & $<0.001$ \\
\hline Cardiovascular related hospitalization & $20(14.2 \%)$ & $84(50.9 \%)$ & $4.87(2.99-7.95)$ & $<0.001$ & $<0.001$ \\
\hline Thrombosis events & $3(2.1 \%)$ & $21(12.7 \%)$ & $6.06(1.81-20.35)$ & 0.001 & 0.009 \\
\hline
\end{tabular}

NS, None Significant.

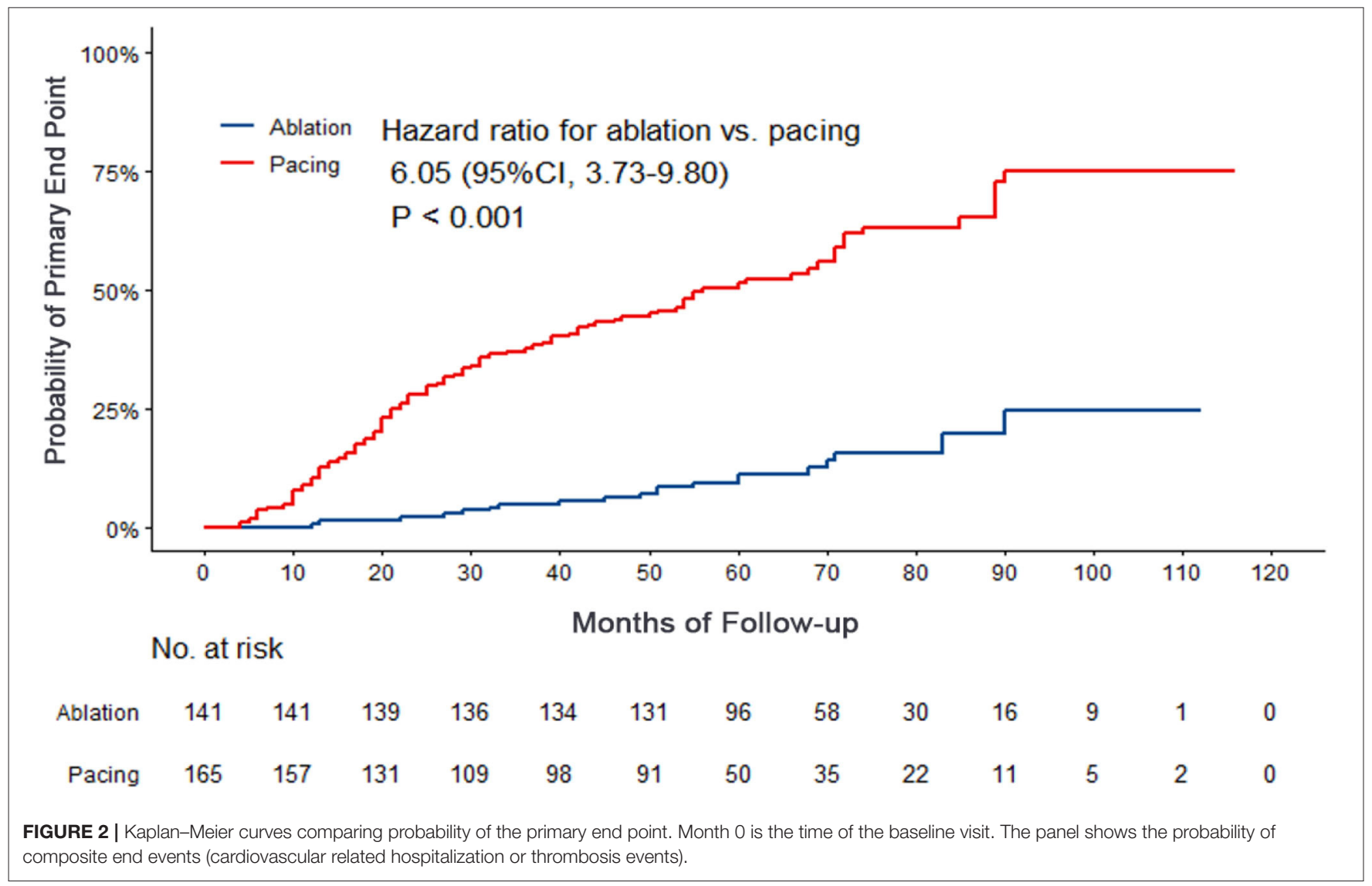

therapy. Furthermore, catheter ablation reduced the progression of atrial fibrillation and heart failure. To our knowledge, this study was the first to compare the effects of catheter ablation and cardiac pacing on the long-term prognosis in TBS patients, TBS patients may be benefit from ablation therapy vs. pacing therapy.

\section{Pacing Therapy in TBS}

Kaplan and Langendorf were the first to describe TBS in 1973 (11). Patients with TBS often suffer from syncope, syndrome, and even sudden death due to a long pause on termination of atrial fibrillation. Cardiac pacing effectively corrects long pauses after atrial arrhythmia to avoid the occurrence of symptoms, and is recommended by guidelines as the primary treatment plan. However, clinical problems related to atrial fibrillation are still unresolved, and related problems caused by pacing are also occurred. A randomized controlled trial conducted by Lau et al. included 385 patients with paroxysmal atrial fibrillation combined with sinus node dysfunction (SSS), and compared the effects of right atrial appendage pacing and right atrial septum pacing on atrial fibrillation (15). The follow-up of the study lasted 3.1 years, and $25.8 \%$ of the patients progressed to persistent atrial fibrillation regardless of the pacing positions and patterns. The Danish Multicenter Randomized trial on single-lead atrial pacing vs. dual-chamber placing in sick sinus syndrome (DANPACE) trial is a randomized controlled trial comparing the effect of single-lead atrial pacemaker (AAIR) pacing or dual chamber pacemaker (DDDR) pacing on long-term prognosis in SSS patients, including 1,348 patients with a follow-up of 5.4 years (16). Of these patients, $25.7 \%$ developed atrial fibrillation. In 


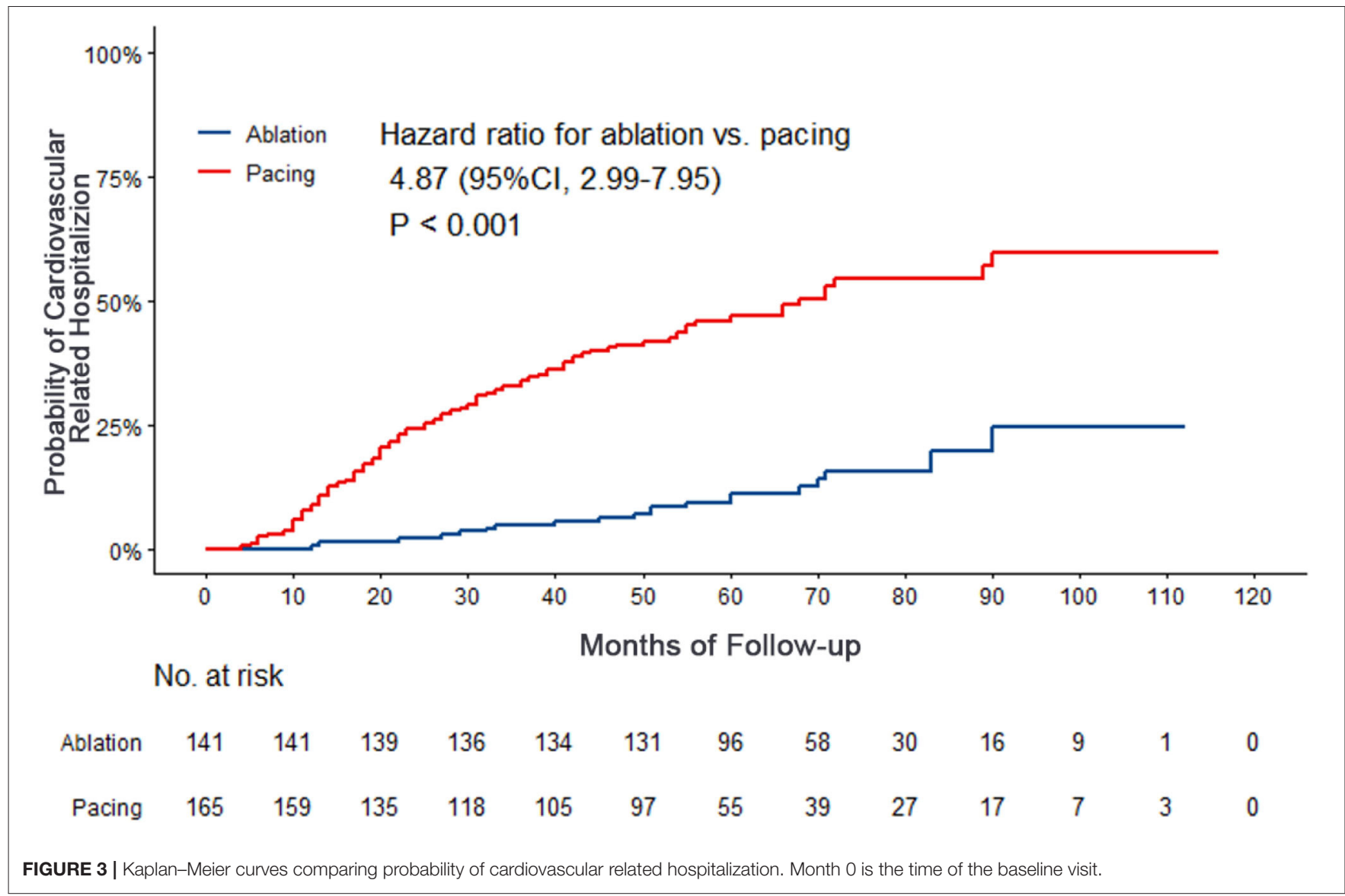

addition, $11.2 \%$ of the patients progressed to chronic atrial fibrillation. In our study, $23.6 \%$ of the patients progressed to persistent atrial fibrillation, which was consistent with the results of the previous study. In addition, thromboembolic events were a main risk for patients with pacemakers. Brandt et al. (16) conducted a registered trial to evaluate the impact of AAI and DDD pacing modes on rehospitalization or stroke in patients with SSS. In the study the incidence of stroke was nearly $10 \%$ regardless of which pacing mode was used. In the present study, the incidence of stroke and peripheral thrombosis was $12.7 \%$. The study conducted by Kristensen et al. (17) showed that atrial fibrillation was an independent risk factor for thrombotic events in patients undergoing pacing (RR: 7.5, 95\% CI: 1.6$36.2, P=0.01$ ). Oral anticoagulants are an effective strategy to prevent thromboembolism. However, in this study, only $16.4 \%$ of our patients received anticoagulation therapy, but these patients had a relatively high $\mathrm{CHA}_{2} \mathrm{DS}_{2}$-VASC score. Insufficient anticoagulation may be one of the reasons for the high risk of thrombotic events. In fact, there are still challenges in requiring strict anticoagulation therapy for these patients. For example, bleeding, pacemaker pocket infections, and patient compliance may be the primary concerns. For patients with TBS undergoing pacemaker implantation, it is inevitable that anticoagulation, rhythm control, and ventricular rate control of atrial fibrillation will need to be addressed again.

\section{Catheter Ablation for Atrial Fibrillation in TBS Patients}

Numerous studies have confirmed that catheter ablation is an effective and safe method for treating paroxysmal atrial fibrillation, with a success rate of $>82 \%$ in $>5$ years (18). A retrospective study conducted by Hada et al. included 65 patients with SSS and atrial fibrillation (SSS-AF). After an average of 1.4 ablations, the patients underwent a 3-year follow-up, showing a success rate of $80.6 \%$ (19). Osaka et al. also conducted a study of catheter ablation in patients with SSS-AF and pacemaker implantation ( $n=51$, followed up for 5 years) (20). The success rate of catheter ablation in the study was $86.3 \%$. Inada et al. (21) performed a study was to define the potential role of successful ablation in patients with TBS. During the 5.8 years (range: 5-8.7 years) follow up, $86 \%$ patients remained free from AF after the last procedure. Only $8 \%$ patients required pacemaker implantation of the study by Chen et al. (22) evaluated the effectiveness and safety of catheter ablation in patients with TBS. Although only 43 patients with TBS were included in the study, 41 (95.3\%) patients, who underwent follow-up for 20 months, did not require pacemaker implantation. Kim et al. (23) conducted a larger sample retrospective study involving 121 patients with TBS and followed up for 20 months. They found that $90.9 \%$ of the patients with TBS did not need pacemaker implantation. In this study, $88.6 \%$ of the patients with TBS 


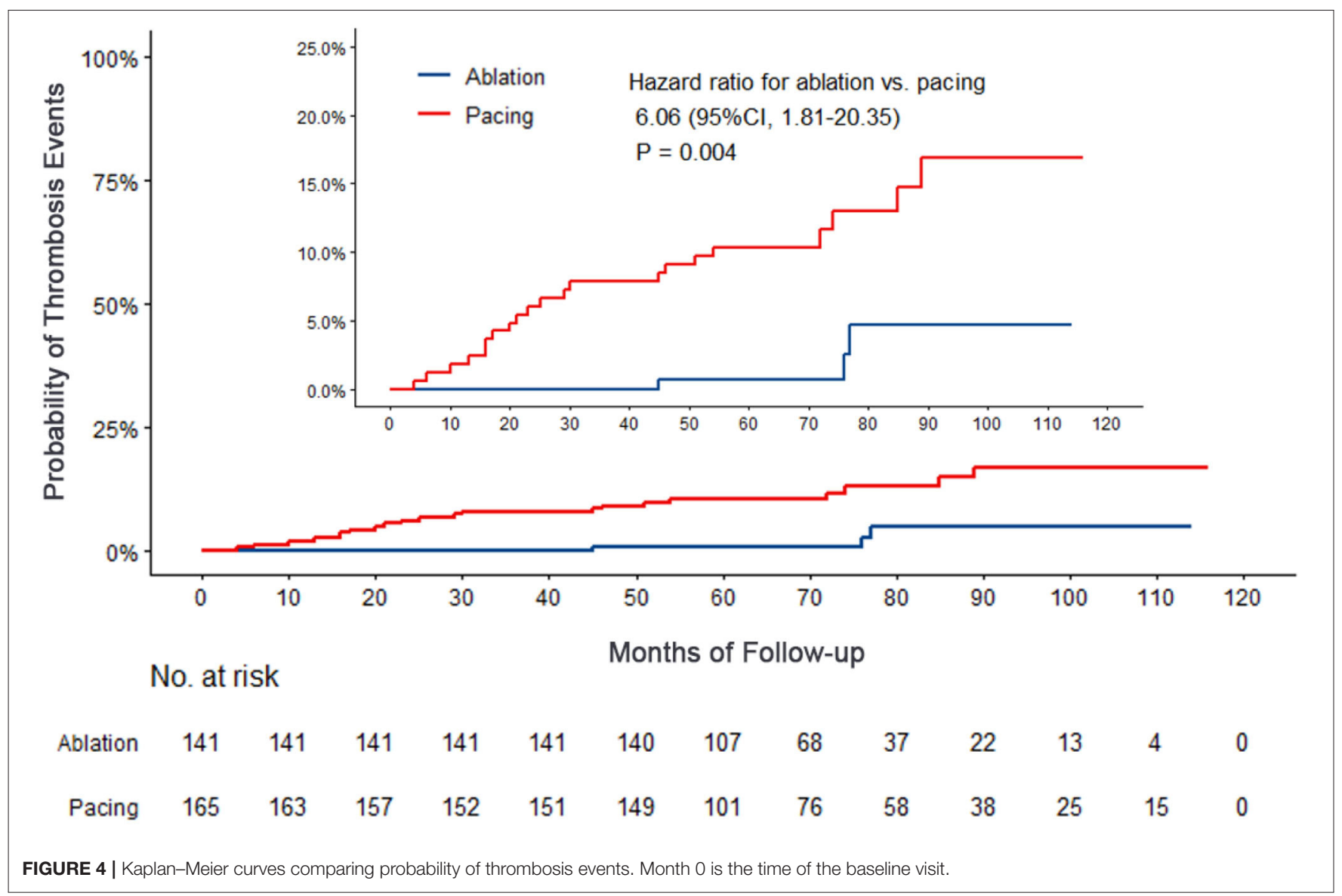

undergoing catheter ablation avoided pacemaker implantation, suggesting that catheter ablation can prevent nearly $90 \%$ of the patients with TBS from requiring an implanted pacemaker.

\section{Should Catheter Ablation Be the First Line Therapy for TBS Patients?}

Cardiovascular related hospitalization, thromboembolic events, and heart failure progression are essential endpoints for evaluating the prognosis of TBS patients. However, few studies have evaluated the hard endpoints of catheter ablation vs. pacing in patients with TBS. Chen et al. (22) showed that catheter ablation significantly reduced the rate of rehospitalization related to atrial fibrillation. However, no significant difference in cardiovascular rehospitalization rate was found, which may be due to the small sample size of that study. One of our previous studies (24) showed that catheter ablation significantly reduced the risk of new strokes in patients with TBS compared with the pacing group $(15.1$ vs. $5.4 \%, P<0.05)$. In this study, after 5.9 years of follow-up, the risk of cardiovascular-related hospitalization and thrombosis in the TBS patients undergoing cardiac pacing was 6.05 -fold higher than that of the patients undergoing catheter ablation (95\% CI: 3.73-9.80, $P<0.001)$. The risk of cardiovascular related hospitalization of the pacing group was 4.87-fold than that of the ablation group (95\% CI: 2.99-7.95, $P<0.001)$, and the thromboembolic event risk of the pacing group was 6.06-fold than that of the ablation group (95\% CI: 1.81-20.35, $P=0.001)$. The long-term outcome data suggested that catheter ablation significantly reduced cardiovascularrelated hospitalizations, strokes, and peripheral thromboembolic events, and also effectively reduced atrial fibrillation burden and heart failure progression. Our findings supported that pacing may be a risk factor for worse prognosis in TBS patients. In this cohort, patients received the RV pacing. Considering the impact of right ventricular pacing on heart function, the results may be bias. Recent years, His bundle pacing (HBP) or left bundle branch pacing can achieve the physiological pacing via directly stimulating the His-Purkinje conduction bundle, which can significantly reduce pacing induced cardiomyopathy (25). Future evidence was needed to verify the results in the TBS patients.

\section{Limitations}

This was a retrospective study, so the clinical evidence level is low, and a prospective randomized controlled study is needed to verify our findings. TBS patients in the pacing group had a higher proportion of anticoagulation but inadequate, suggesting that an increase in anticoagulation rate may effectively reduce the incidence of thromboembolic events in the pacing group. Lower use of anticoagulation in the entire group may limit the applicability of the data. Adequate use of anticoagulation in TBS patients may reduce the difference of the prognosis between the 
two therapy strategies. In our series these patients had relatively normal left atrial size. This data may not be applicable to patients with moderate or severely dilated left atria. Additionally, there may have been a selection bias during the selection of treatment strategies in this retrospective study.

\section{DATA AVAILABILITY STATEMENT}

The original contributions presented in the study are included in the article/supplementary material, further inquiries can be directed to the corresponding author/s.

\section{ETHICS STATEMENT}

The studies involving human participants were reviewed and approved by the ethics committee of First Affiliated Hospital of Dalian Medical University. Written informed consent for

\section{REFERENCES}

1. Clementy N, Andrade JG, Babuty D, Roy D. Unusual tachycardia-bradycardia syndrome during atrial fibrillation: what is the mechanism? Circ Arrhythm Electrophysiol. (2013) 6:e42-6. doi: 10.1161/CIRCEP.113.000151

2. Tse G, Liu T, Li KH, Laxton V, Wong AO, Chan YW, et al. Tachycardia-bradycardia syndrome: electrophysiological mechanisms and future therapeutic approaches (Review). Int J Mol Med. (2017) 39:519-26. doi: 10.3892/ijmm.2017.2877

3. Lo LW, Tuan TC, Tai CT, Lin YJ, Chang SL, Chen SA. Incessant wide QRS tachycardia after pulmonary vein isolation and pacemaker implantation in a patient with tachycardia-bradycardia syndrome. J Cardiovasc Electrophysiol. (2007) 18:783-4. doi: 10.1111/j.1540-8167.2007.00825.x

4. Calkins H, Hindricks G, Cappato R, Kim YH, Saad EB, Aguinaga L, et al. 2017 HRS/EHRA/ECAS/APHRS/SOLAECE expert consensus statement on catheter and surgical ablation of atri al fibrillation: executive summary. Heart Rhythm. (2017) 14:e445-94. doi: 10.1016/j.hrthm.2017.07.009

5. Kirchhof P, Benussi S, Kotecha D, Ahlsson A, Atar D, Casadei B, et al. 2016 ESC guidelines for the management of atrial fibrillation developed in collaboration with EACTS. Eur Heart J. (2016) 37:2893962. doi: 10.1093/eurheartj/ehw210

6. Flaker GC, Blackshear JL, McBride R, Kronmal RA, Halperin JL, Hart RG. Antiarrhythmic drug therapy and cardiac mortality in atrial fibrillation. The Stroke Prevention in At rial Fibrillation Investigators. J Am Coll Cardiol. (1992) 20:527-32. doi: 10.1016/0735-1097(92)90003-6

7. Elvan A, Wylie K, Zipes DP. Pacing-induced chronic atrial fibrillation impairs sinus node function in dogs. Electrophysiological remodeling. Circulation. (1996) 94:2953-60. doi: 10.1161/01.CIR.94.11.2953

8. Sanders P, Morton JB, Kistler PM, Vohra JK, Kalman JM, Sparks PB. Reversal of atrial mechanical dysfunction after cardioversion of atrial fibrillation: implications fo $r$ the mechanisms of tachycardia-mediated atrial cardiomyopathy. Circulation. (2003) 108:1976-84. doi: 10.1161/01.CIR.0000091408.45747.04

9. Shinbane JS, Wood MA, Jensen DN, Ellenbogen KA, Fitzpatrick AP, Scheinman MM. Tachycardia-induced cardiomyopathy: a review of animal models and clinical studies. J Am Coll Cardiol. (1997) 29:709-15. doi: 10.1016/S0735-1097(96)00592-X

10. Petersen LDD, Christiansen MK, Pedersen LN, Nielsen JC, Broendberg $\mathrm{AK}$, Jensen HK. Implantable cardioverter-defibrillator therapy and devicerelated complications in young patients wit $\mathrm{h}$ inherited cardiomyopathies or channelopathies: a 17-year cohort study. Europace. (2018) 20:184955. doi: 10.1093/europace/euy081

11. Kaplan BM, Langendorf R, Lev M, Pick A. Tachycardia-bradycardia syndrome (so-called "sick sinus syndrome"). Pathology, mechanisms and participation was not required for this study in accordance with the national legislation and the institutional requirements.

\section{AUTHOR CONTRIBUTIONS}

YX, LG, and XYi contributed to conception and design of the study. YW, ZW, and MY organized the database. XYu and XX performed the statistical analysis. YW wrote the first draft of the manuscript. RZ wrote sections of the manuscript. All authors contributed to manuscript revision, read, and approved the submitted version.

\section{FUNDING}

This research was supported by National Natural Science Foundation of China (No. 81800294) and Dalian High-level Talent Innovation Support Program (No. 2018R09).

treatment. Am J Cardiol. (1973) 31:497-508. doi: 10.1016/0002-9149(73) 90302-0

12. Waleed KB, Guan X, Li X, Yang Y, Wang Z, Yin X, et al. Atrial fibrillation is related to lower incidence of deep venous thrombosis in patients with pulmonar y embolism. J Thorac Dis. (2018) 10:147682. doi: $10.21037 /$ jtd.2018.01.177

13. Dong YX, Powell BD, Asirvatham SJ, Friedman PA, Rea RF, Webster TL, et al. Left ventricular lead position for cardiac resynchronization: a comprehensive cinegraphic, echocardio graphic, clinical, and survival analysis. Europace. (2012) 14:1139-47. doi: 10.1093/europace/eus045

14. Lip GY, Nieuwlaat R, Pisters R, Lane DA, Crijns HJ. Refining clinical risk stratification for predicting stroke and thromboembolism in atrial fibrillatio $\mathrm{n}$ using a novel risk factor-based approach: the euro heart survey on atrial fibrillation. Chest. (2010) 137:263-72. doi: 10.1378/chest.09-1584

15. Lau CP, Tachapong N, Wang CC, Wang JF, Abe H, Kong CW, et al. Prospective randomized study to assess the efficacy of site and rate of atrial pacing on longterm pr ogression of atrial fibrillation in sick sinus syndrome: septal pacing for atrial fibrillation suppr ession evaluation (SAFE) study. Circulation. (2013) 128:687-93. doi: 10.1161/CIRCULATIONAHA.113.001644

16. Brandt NH, Kirkfeldt RE, Nielsen JC, Mortensen LS, Jensen GVH, Johansen JB, et al. Single lead atrial vs. dual chamber pacing in sick sinus syndrome: extended register-based follow-up in the DANPACE trial. Europace. (2017) 19:1981-7. doi: 10.1093/europace/euw364

17. Kristensen L, Nielsen JC, Mortensen PT, Pedersen OL, Pedersen AK, Andersen HR. Incidence of atrial fibrillation and thromboembolism in a randomised trial of atrial versus dual cham ber pacing in 177 patients with sick sinus syndrome. Heart. (2004) 90:661-6. doi: 10.1136/hrt.2003.016063

18. Ganesan AN, Shipp NJ, Brooks AG, Kuklik P, Lau DH, Lim HS, et al. Long-term outcomes of catheter ablation of atrial fibrillation: a systematic review and meta-analysis. J Am Heart Assoc. (2013) 2:e004549. doi: 10.1161/JAHA.112.004549

19. Hada M, Miyazaki S, Kajiyama T, Yamaguchi M, Kusa S, Nakamura H, et al. Catheter ablation of paroxysmal atrial fibrillation in patients with sick sinus syndrome. Heart Vessels. (2019) 34:503-8. doi: 10.1007/s00380-0181256-8

20. Osaka Y, Takigawa M, Takahashi A, Kuwahara T, Okubo K, Takahashi Y, et al. The proportion of asymptomatic recurrence after catheter ablation of atrial fibrillation in patients with a pacemaker for sick sinus syndrome. Indian Pacing Electrophysiol J. (2017) 17:125-31. doi: 10.1016/j.ipej.2017. 07.009

21. Inada K, Yamane T, Tokutake K, Yokoyama K, Mishima T, Hioki M, et al. The role of successful catheter ablation in patients with paroxysmal atrial fibrillation and prolonge d sinus pauses: outcome during a 5-year follow-up. Europace. (2014) 16:208-13. doi: 10.1093/europace/eut159 
22. Chen YW, Bai R, Lin T, Salim M, Sang CH, Long DY, et al. Pacing or ablation: which is better for paroxysmal atrial fibrillation-related tachycardia-bradycardi a syndrome? Pacing Clin Electrophysiol. (2014) 37:403-11. doi: 10.1111/pace.12340

23. Kim DH, Choi JI, Lee KN, Ahn J, Roh SY, Lee DI, et al. Longterm clinical outcomes of catheter ablation in patients with atrial fibrillation predisposing to tachycardia-bradycardia syndrome: a long pause predicts implantation of a permanent pacemaker. BMC Cardiovasc Disord. (2018) 18:106. doi: 10.1186/s12872-0180834-0

24. Zhang S, Yang Y, Xia Y, Gao L, Zhang X, Tse G, et al. Long-term effect of catheter ablation on tachycardia-bradycardia syndrome: evidenced by 10 years foll ow up. Acta Cardiol. (2020) 75:537-543. doi: 10.1080/00015385.2019. 1630055

25. Abdelrahman M, Subzposh FA, Beer D, Durr B, Naperkowski A, Sun H, et al. Clinical outcomes of his bundle pacing compared to right ventricular pacing. J Am Coll Cardiol. (2018) 71:2319-30. doi: 10.1016/j.jacc.2018 02.048

Conflict of Interest: The authors declare that the research was conducted in the absence of any commercial or financial relationships that could be construed as a potential conflict of interest.

The handling editor declared a past co-authorship with one of the authors YX.

Copyright (c) 2021 Zhang, Wang, Yang, Yang, Wang, Yin, Dong, Yu, Xiao, Gao and Xia. This is an open-access article distributed under the terms of the Creative Commons Attribution License (CC BY). The use, distribution or reproduction in other forums is permitted, provided the original author(s) and the copyright owner (s) are credited and that the original publication in this journal is cited, in accordance with accepted academic practice. No use, distribution or reproduction is permitted which does not comply with these terms. 